\title{
ESTIMACIÓN DE LA DEMANDA DE USO DE AUTOS PARTICULARES EN LA ZONA METROPOLITANA DEL VALLE DE MÉXICO: UN ANÁLISIS TOBIT
}

Rubén Santos-Pérez

(Recibido: abril, 2015/Aceptado: agosto/2015)

\section{Resumen}

En este trabajo se analiza el efecto del ingreso de los hogares, así como de variables socioeconómicas y geográficas sobre la demanda de uso de autos particulares en la Zona Metropolitana del Valle de México. Se emplea un modelo Tobit a fin de captar los efectos de los hogares que demandan el uso de autos y de aquellos hogares que podrían ingresar al mercado. Los resultados muestran que la variable más importante en la determinación del uso de vehículos por parte de los hogares es el ingreso. Además, nuestro estudio muestra que variables como la edad, número de personas ocupadas y distancia, afectan significativamente a la demanda de uso de autos y que el efecto más importante sobre el uso de los mismos se debe a los nuevos consumidores que ingresan al mercado.

Palabras clave: ingreso de hogares, modelo tobit, Valle de México, autos. Clasificación JEL: C24, C51, D12, R21, R41.

\begin{abstract}
In this work the effect of household income is analyzed as well as socioeconomic and geographical demand for use of private cars in the Metropolitan
\end{abstract}

Consultor y profesor en la Escuela Superior de Economía del Instituto Politécnico Nacional. 
Zone of the Mexican Valley variables. A Tobit model to capture the effects of households that demand the use of cars and homes that could be used to enter the market. The results show that the most important in determining the variable vehicle use by households is income. In addition, our study shows that variables such as age, number of employed people and distance, significantly affect the demand for car use and that the most important on the use of the same is due to the new consumers entering the market.

Keywords: household income, tobit, Valley of Mexico, cars

JEL classification: C24, C51, D12, R21, R41.

\section{Introducción}

La movilidad urbana es un elemento trascendental en la Zona Metropolitana del Valle de México (ZMVM), ya que ésta influye directamente sobre el bienestar social, el desarrollo económico y la competitividad. A su vez, sobre dicha movilidad, inciden la eficiencia del transporte público, la disponibilidad de infraestructura y vialidades, así como la dimensión del parque vehicular.

Respecto a este último punto, la flota vehicular en la ZMVM pasó de poco más de 2 millones de unidades en 1990 (García, 2010), a poco más de 5 millones en 2012 (Gobierno del Distrito Federal, 2013); sin embargo, ante una oferta fija de infraestructura (carreteras, libramientos, pasos a desnivel, distribuidores viales, servicios de tránsito, entre otros), el incremento en el parque vehicular se ha traducido en una mayor congestión vial y en un aumento de la contaminación ambiental.

En el caso particular del Distrito Federal, el crecimiento del parque vehicular dio como resultado que la velocidad promedio de autos se redujera a poco más de la mitad de 1990 a 2007, al pasar de 38.5 a 17 kilómetros por hora, respectivamente. Además, la velocidad promedio de los autos en 2013, decayó notablemente hasta ubicarse en 12 kilómetros por hora, Instituto de Políticas para el Transporte y el Desarrollo (ITDP), 2014.

La mala movilidad ocasionada por el congestionamiento vehicular produce una pérdida diaria de 3.3 millones de horas-hombre en la ZMVM, equivalentes a 33 mil millones de pesos anuales (IMCO, 2012). En este tenor, algunos autores como Márquez y Pradilla (2007), afirman que el patrón de 
movilidad en la ZMVM se ha inclinado hacia el predominio del automóvil, emulando el camino de las ciudades estadounidenses, por lo que sugieren redefinir el patrón de movilidad de la ZMVM, fundada en una estrategia que priorice un transporte colectivo eficiente, con sustentabilidad social y ambiental.

En la misma dirección, el Instituto de Políticas para el Transporte y el Desarrollo de México (2012a) y (2012b) ha planteado una serie de estrategias para reducir el uso del automóvil en las principales metrópolis del país. Sobre esto, Islas (2000), agrega que las características de la población deben de ser el punto de partida para la definición de la política de transporte y, en general, de movilidad para toda la ZMVM. Es por ello, que contar con estudios que tomen en cuenta las características de la población pueden abonar al diseño de una política de movilidad para la ZMVM.

Acorde con lo anterior, el objetivo del presente documento consiste en analizar los determinantes individuales de la demanda de transporte privado; específicamente, la demanda de uso de autos particulares en la ZMVM, a partir de un modelo Tobit; para que con base en ello se puedan derivar algunas recomendaciones de política pública en la materia.

Con ese propósito, el presente trabajo se divide en cinco partes, siendo la primera la presente introducción; la segunda parte, la referida a la revisión de la literatura. En la tercera parte se expone la metodología econométrica en donde se explica la distinción entre distribuciones truncadas y censuradas y cómo, a partir de dicho análisis, se puede derivar la construcción del modelo Tobit. La cuarta parte se refiere a la estimación del modelo Tobit para determinar las variables relevantes que afectan la demanda de uso de automóviles en la ZMVM, en donde se describe la base de datos que se emplea en este estudio y se presentan y se explican los principales resultados obtenidos a partir de la estimación del modelo Tobit. Por último, en la quinta parte se presentan las conclusiones de nuestro estudio.

\section{Revisión de la literatura}

La demanda del uso del automóvil es un tema que ha recibido bastante atención por parte de los economistas desde hace varios años; el estudio pionero de Benett (1967) se enfocó en ese sentido. Este autor realizó un estudio completo de la demanda de uso de automóviles en Estados Unidos 
para el periodo 1955-1957, para lo cual empleó la metodología Tobit. En su investigación utilizó la depreciación de los vehículos como una variable proxy del consumo de los mismos, mientras que variables como el ingreso y algunas características sociodemográficas de los hogares las tomó como regresores. Sus resultados arrojaron que la demanda de uso de autos crecía con el ingreso, pero a una tasa menor para los hogares con altos ingresos, en relación a las unidades demandadas por los hogares de bajos ingresos, cuya tasa de crecimiento era mayor. Benett (1967) también encontró que el gasto de los hogares aumentaba con el número de adultos presentes en dicha unidad familiar; asimismo, observó que la demanda de uso de autos disminuía a medida que aumentaba el número de menores de edad en el hogar. De manera análoga, encontró que la demanda se reducía a medida que el jefe de hogar se aproximaba a su edad de jubilación; por último, observó que los hogares que se localizaban en los lugares alejados de los centros de las ciudades de Nueva York y Chicago tenían un mayor gasto en relación a los que habitaban relativamente cerca de las zonas centrales de dichas ciudades, debido a que los primeros tenían que realizar un mayor desplazamiento.

Posteriormente, los estudios para analizar la demanda de uso de autos se inclinó en mayor medida a la aplicación de modelos de decisión múltiple, en donde el enfoque principal consistía en analizar los factores que determinaban la elección del modo de transporte para desplazarse a su lugar de trabajo. Para lograr lo anterior, se emplearon dos grandes grupos de metodologías; en el primer grupo se encontraban aquellas conformadas por los modelos probit y logit multivariados; las cuales se retomaron en los trabajos de Ben-Akiva y Lerman (1975) y en el de Donnea (1971); mientras que el segundo grupo, conformado por los modelos logit multivariados anidados, destacaron los trabajos de De Palma y Rochat (2000), Thobani (1984) y Train (1980). Cabe señalar que en ambos grupos se empleó información a nivel de hogar o de individuo para analizar las variables que determinaban la demanda de uso de auto o de transporte público.

En este contexto, los trabajos de Ming-Chu (1994) y el de Nolan (2002) retomaron el enfoque de Benett (1967), al emplear el modelo Tobit para analizar la demanda de uso de autos particulares y de transporte público por parte de los hogares, basándose en las características de las unidades familiares.

En particular, el presente trabajo se basa en el estudio de Nolan (2002), quien a partir de información a nivel de hogar obtuvo los determinantes 
sociodemográficos de las decisiones de transporte de los hogares de Irlanda, para lo cual empleó la Encuesta de Presupuesto de los Hogares Irlandeses correspondiente al periodo 1994/1995. Este autor empleó el gasto en gasolina como una variable proxy de la demanda de uso del automóvil de los hogares; además, utilizó el gasto total per cápita como proxy del ingreso total per cápita del hogar; asimismo, recurrió a variables sociodemográficas como el número de adultos en el hogar y el número de niños; también incorporó características del jefe del hogar, como género, edad y escolaridad; por último, agregó variables que permitían identificar la distancia aproximada de desplazamiento de los integrantes de los hogares.

Los principales resultados del estudio de Nolan (2002) arrojaron que el ingreso es una de las variables más importantes para explicar las diferencias en las decisiones de transporte de los hogares; además, éstos señalan que a medida que aumenta el número de adultos en el hogar se incrementa la demanda de uso de vehículos; y que el género del jefe del hogar es una variable significativa que explica en gran medida el uso del auto, lo mismo que el gasto en transporte público, en donde el gasto es mayor para los hogares dirigidos por un hombre.

Cabe precisar, que el efecto de la edad del jefe del hogar es consistente con lo esperado, respecto a que los hogares jóvenes tienen mayor movilidad, debido a que desarrollan más actividades que los hogares con integrantes de mayor edad.

Para el caso específico de México existen pocos estudios de este tipo, de ahí la relevancia del presente trabajo. Entre los estudios más importantes se puede citar el de Del Muro (2006) quien analiza los determinantes de las decisiones de transporte de los hogares en México, para lo cual estima la demanda de transporte público, como privado por parte de los mismos. Desde el punto de vista del transporte privado, dicho autor estima la probabilidad de que un hogar cuente con un automóvil y al mismo tiempo estima la demanda de uso del mismo, para lo cual recurre al consumo de gasolina como proxy de esa demanda. A su vez, el análisis de la demanda de transporte público la realiza a partir del uso de los servicios de camión, de taxi y de metro. Del Muro (2006), al igual que Nolan (2002), emplea un modelo Tobit para estimar la demanda de transporte tanto público como privado.

Los principales resultados del estudio de Del Muro (2006) muestran que la variable más importante en la elección de los medios de transporte es el ingreso. Sus estimaciones arrojan que el uso del automóvil, así como la 
demanda de servicios de camión y taxi en México se comporta como bienes necesarios. El trabajo de Del Muro (2006), al tener un alcance nacional, permite concluir que los hogares de la región noroeste de México presentan una mayor probabilidad de poseer un auto y en contra parte, las unidades familiares de la región sureste tienen una menor probabilidad.

Adicionalmente, dicho autor concluye que los jefes de hogar hombres usan en mayor medida el automóvil en relación a sus pares mujeres. Lo mismo que los jefes de hogar casados en relación a los jefes solteros, acentuándose en las regiones sureste y centro occidente, respecto a la primera característica y en las regiones noroeste y centro sur para la segunda. Por último, los resultados del estudio de Del Muro (2006) muestran que las mujeres presentan una mayor disponibilidad a usar el transporte público en relación a los hombres y que a medida que aumenta la escolaridad con la que cuentan los jefes de hogar, su demanda de uso de automóvil también se incrementa.

\section{Metodología econométrica}

El truncamiento y el censuramiento de los datos estadísticos son dos problemas que ocurren con mucha frecuencia en el análisis econométrico aplicado. Con respecto a la segunda característica, los modelos de regresión convencionales no toman en cuenta la diferencia cualitativa entre las observaciones censuradas y las no censuradas, lo cual arroja valores estimados que son sesgados, inconsistentes e ineficientes.

Para tomar en cuenta la presencia del censuramiento de la variable dependiente en el análisis de regresión, el premio Nobel de Economía James Tobin, propuso en 1958 el modelo Tobit como una alternativa para tratar este tipo de variables. Su popularidad se basa en que mediante su uso se puede aprovechar completamente la información contenida en los datos (incluyendo observaciones con valores cero para la variable dependiente). El modelo aumentó todavía más su popularidad con el estudio que McDonald y Moffit elaboraron en 1980, en donde ellos sugirieron una útil descomposición de los coeficientes Tobit estimados.

$\mathrm{Al}$ respecto, dicha metodología se acopla perfectamente a las necesidades del presente estudio, ya que una parte de la población no cuenta con auto y por ello su demanda de auto privado, medida por su consumo de gasolina, 
es cero. Mientras que por otro lado, un segmento de la población total se caracteriza por tener una demanda positiva de dicho bien.

\subsection{Distribuciones censuradas y truncadas}

Como se ha referido previamente, en el análisis de variables dependientes limitadas, existen dos casos de interés que son el censuramiento y el truncamiento.

De acuerdo con Smith (2005), el truncamiento ocurre cuando algunos valores de una variable continua subyacente simplemente no se observan. Por ejemplo, cuando el gobierno recolecta solamente datos de las empresas que se encuentran por encima de algún tamaño, con la finalidad de minimizar la carga regulatoria. ${ }^{1}$ Por otro lado, el censuramiento ocurre cuando existe una variable continua subyacente, pero para un rango de valores de la variable se codifica en un número, agrupando la masa de probabilidad en un punto (Smith, 2005). Un ejemplo es la cantidad de dinero que gastan las personas en cerveza en un determinado periodo; para algunos individuos, principalmente mayores de 18 años, esta variable toma una gran cantidad de valores diferentes de cero; mientras que para otra parte importante de la población, la cantidad gastada en cerveza es cero (Villezca y Moreno, 2000).

La diferencia fundamental entre el censuramiento y el truncamiento es que en el primero, las observaciones están presentes en los datos que se tienen, y se sabe que se han censurado; a su vez, en el caso del truncamiento, las observaciones simplemente faltan y su naturaleza generalmente se revela por fuentes externas. ${ }^{2}$

Por lo general, el censuramiento en un rango de valores, para la variable de interés, introduce una distorsión en los resultados estadísticos convencionales, los cuales son similares a los del truncamiento, básicamente es un problema de sesgo por variables omitidas (Wooldridge, 2010). Adicionalmente, el censuramiento es un problema más común en estudios recientes.

${ }^{1}$ Otro caso se presenta en algunos estudios sobre los ingresos, en donde el análisis se puede enfocar en los ingresos que se encuentran por encima o por debajo de alguna línea de riqueza; por ejemplo, los ingresos de las personas que ganan menos de tres salarios mínimos.

2 A diferencia del truncamiento, el censuramiento es esencialmente un defecto de la muestra. Si los datos no estuvieran censurados, los datos serían una muestra representativa de la población de interés (Greene, 2012). 
Cabe señalar que desde un punto de vista metodológico, se debe analizar en primera instancia el problema del truncamiento, ya que proporciona la mayoría de las herramientas teóricas que se requieren para el estudio del censuramiento.

El cálculo de los momentos de una distribución truncada se puede simplificar si se emplea la función generadora de momentos de una variable normal truncada: ${ }^{3}$

$$
m_{Y} \mid Y_{>a}(t)=\frac{1-\Phi(\omega)}{1-\Phi(\alpha)} e^{\mu t+\frac{1}{2} \sigma^{2} t^{2}}
$$

A partir de ello, y siendo a es una constante, entonces:

$$
\begin{gathered}
E[y \mid y>a]=\mu+\sigma \lambda(\alpha) \\
\operatorname{Var}[y \mid y>a]=\sigma^{2}[1-\delta(\alpha)]
\end{gathered}
$$

Donde $\alpha=\frac{\alpha-\mu}{\sigma}, \mathrm{y} \varphi(\alpha)$ es la función de densidad de una variable aleatoria normal estándar. Además,

$$
\begin{aligned}
& \lambda(\alpha)=\frac{\Phi(\alpha)}{1-\Phi(\alpha)} \text { si el truncamiento es } y>a \\
& \lambda(\alpha)=\frac{-\Phi(\alpha)}{\Phi(\alpha)} \text { si el truncamiento es } y<a
\end{aligned}
$$

Y

$$
\delta(\alpha)=\lambda(\alpha)[\lambda(\alpha)-\alpha] \text { si el truncamiento es } y>a
$$

\subsection{Distribuciones censuradas}

Específicamente, el problema del censuramiento ocurre con bastante frecuencia en los datos de tipo microeconómico; en particular, cuando se censura la variable dependiente de un modelo de regresión lineal, los

\footnotetext{
${ }^{3}$ Una de las características del presente documento es la utilización de funciones generadoras de momentos para la obtención de los momentos de una variable aleatoria truncada.
} 
valores que se encuentran en un cierto rango se transforman (o se reportan) como un solo valor (Greene, 2012).

La teoría relevante para analizar la distribución de una variable censurada es similar a la de las variables truncadas. El punto de censuramiento puede darse en cualquier valor, aunque un caso que más se ha estudiado y aplicado es cuando el punto de censuramiento ocurre en cero.

De acuerdo con Greene (2012), en una distribución truncada, sólo la parte de la distribución que se encuentra por encima de y = a es relevante para los cálculos; para que la distribución integre uno, se escala la probabilidad de que una observación en la población no truncada caiga en el rango que interesa. No obstante, cuando los datos están censurados, la distribución que se aplica a la muestra es una mezcla de una distribución continua y una distribución discreta.

Si se define una nueva variable aleatoria y, que es una transformación de la original, $\mathrm{y}^{*}, 4$ la cual se supone que da origen a la distribución subyacente que se censura; es decir, se define:

$$
\begin{gathered}
y=a \text { si } y * \leq a \\
y=y * \text { si } y *>a
\end{gathered}
$$

Por lo que la distribución que se aplica si $\mathrm{y}^{*} \leq a$ es:

$$
\operatorname{Pr}(y=a)=\operatorname{Pr}(y * \leq a)=\Phi\left(\frac{a-\mu}{\sigma}\right)
$$

Y si $\mathrm{y}^{*}>\mathrm{a}$, entonces $\mathrm{y}$ tiene la densidad de $\mathrm{y}^{*}$.

Así, la distribución es una mezcla de partes continuas y discretas. La probabilidad total es uno, como se requiere, pero en lugar de escalar la parte discreta, simplemente se le asigna la probabilidad total de la región censurada al punto de censuramiento, en este caso, $a$.

En este contexto, se pueden obtener los moment os de la variable normal censurada, los cuales están dados por:

$$
E[y]=\Phi a+(1-\Phi)(\mu+\sigma \lambda)
$$

$\mathrm{y}$

$$
E[y]=\Phi a+(1-\Phi)(\mu+\sigma \lambda)
$$

\footnotetext{
${ }^{4}$ A esta variable se le conoce como una variable latente, porque no se puede observar directamente, sino indirectamente a través de otras variables. 
Y

$$
\text { donde } \Phi\left(\frac{a-\mu}{\sigma}\right)=\Phi(\alpha)=\operatorname{Pr}(y * \leq a)=\Phi, \lambda=\frac{\Phi}{1-\Phi}
$$

$$
\delta=\lambda^{2}-\lambda_{\alpha}
$$

\subsection{El modelo Tobit}

Como ya se ha referido, el modelo Tobit fue sugerido por James Tobin en 1958, quien lo empleó para analizar los gastos de los hogares de Estados Unidos en bienes durables, tomando en cuenta que éstos no podían ser negativos. Por su parte, Arthur Goldberger (1964) se refirió a este modelo como un modelo Tobit, debido a su similitud con los modelos Probit. El modelo se ha generalizado de muchas formas, desde el estudio de Amemiya (1984). Los economistas también se refieren a estas generalizaciones como modelos Tobit, las cuales pueden considerarse como un caso especial de un modelo de regresión censurado.

El modelo Tobit se emplea en los casos en los que la variable dependiente es continua, pero su rango puede estar restringido. Esto ocurre con mayor frecuencia cuando la variable dependiente es cero para una parte importante de la población, pero positiva (con muchos resultados diferentes) para el resto de la misma (Cabrer et al., 2001). El ejemplo clásico se refiere a los gastos de las familias sobre un conjunto de bienes, en donde una parte significativa del conjunto de familias no consumen dichos bienes (cero consumo). Algunos otros ejemplos que han aparecido en la literatura, de acuerdo con Greene (2012), son: el número de horas que labora una mujer que se encuentra en la fuerza de trabajo; el número de arrestos después de salir de prisión; el gasto de los hogares en varios grupos de bienes; los gastos en vacaciones, entre otros; donde se analiza una variable dependiente que es cero para una fracción significativa de las observaciones. ${ }^{5}$

\section{Implicaciones del censuramiento en el análisis de regresión}

De acuerdo con Wooldridge (2010), si se emplea el método de mínimos cuadrados ordinarios (MCO), cuando la variable dependiente se encuentra

s De acuerdo con Villezca y Moreno (2000), los métodos de regresión convencional no toman en cuenta la diferencia cualitativa entre las observaciones límite (cero) y las observaciones no límite (continuas). 
censurada en cero, el modelo estimado podría arrojar estimaciones negativas, equivalentes a predicciones negativas para la variable dependiente, lo cual es similar al caso del modelo de probabilidad lineal, cuando se trabaja con variables binarias.

Ante esto, una solución que podría pensarse consistiría en utilizar solamente la parte de la muestra que incluya las observaciones no restringidas, pero aun así los estimadores de MCO darán estimadores sesgados. Para resolver el problema anterior, se propone el modelo Tobit, el cual toma en cuenta el punto de censuramiento y arroja estimadores insesgados y consistentes.

\subsection{Formulación matemática del modelo Tobit}

El modelo que se basa en una distribución normal censurada en cero se llama modelo de regresión censurado o modelo Tobit. El modelo se formula como un modelo de regresión censurado en $\mathrm{y}=0$, tal como se muestra a continuación:

$$
\begin{array}{lll}
y_{i}^{*}=x_{i}^{\prime} \beta+u_{i} & \\
y_{i}=0 & \text { si } & y_{i}^{*} \leq 0 \\
y_{i}=0 & \text { si } & y_{i}^{*}>0
\end{array}
$$

éste también se puede expresar como:

$$
y_{i}=\max \left(0, y_{i}^{*}\right.
$$

y se agrega el supuesto de normalidad para $u_{i}$, es decir:

$$
u_{i} \mid x_{i} \sim N\left[0, \sigma^{2}\right]
$$

Dado que el modelo Tobit es un caso especial del modelo censurado, entonces el valor esperado de la variable dependiente se puede expresar de la siguiente manera:

$$
E\left[y_{i} \mid x_{i}\right]=\Phi\left(\frac{a-x_{i}^{\prime} \beta}{\sigma}\right) a+\left(1-\Phi\left[x_{i}^{\prime} \beta+\sigma \frac{\Phi\left(\frac{a-x_{i}^{\prime} \beta}{\sigma}\right)}{1-\Phi\left(\frac{a-x_{i}^{\prime} \beta}{\sigma}\right)}\right]\right.
$$


Para ello se recurre a la media de una variable aleatoria censurada por debajo de un valor $a$, en donde $a=0$; con esto se tiene que: 6

$$
E\left[y_{i} \mid x_{i}\right]=\Phi\left(\frac{x_{i}^{\prime} \beta}{\sigma}\right)\left(x_{i}^{\prime} \beta+\sigma \lambda_{i}\right.
$$

o bien,

$$
E\left[y_{i} \mid x_{i}\right]=\Phi_{i} x_{i}^{\prime} \beta+\sigma \phi_{i}
$$

en donde:

$$
\lambda_{i}=\frac{\phi\left(\frac{0-x_{i}^{\prime} \beta}{\sigma}\right)}{1-\Phi\left(\frac{0-x_{i}^{\prime} \beta}{\sigma}\right)}=\frac{\phi\left(\frac{x_{i}^{\prime} \beta}{\sigma}\right)}{\Phi\left(\frac{x_{i}^{\prime} \beta}{\sigma}\right)} y \Phi_{i}=\Phi\left(\frac{x_{i}^{\prime} \beta}{\sigma}\right)
$$

además,

$$
\operatorname{Pr}\left(y_{i}>0 \mid x_{i}\right)=\operatorname{Pr}\left(x_{i}^{\prime} \beta+u_{i}>0\right)=\operatorname{Pr}\left(u_{i}<x_{i}^{\prime} \beta\right)=\Phi\left(\frac{x_{i}^{\prime} \beta}{\sigma}\right)
$$

Por último, se debe subrayar que el supuesto de normalidad es fundamental en este modelo; algunas veces la variable dependiente se puede transformar (tomando logaritmos) para que el supuesto de normalidad resulte más factible (Smith, 2005).

\subsection{Efectos marginales en el modelo Tobit}

A diferencia de los coeficientes del modelo lineal, los coeficientes del modelo Tobit no tienen una interpretación directa, por lo que se requiere del cálculo de los efectos marginales del segundo modelo. ${ }^{7}$

\footnotetext{
- Cabe destacar que la estimación de $\Phi\left(\frac{x_{i} \beta}{\sigma}\right)$, que aparece en el modelo Tobit, se puede obtener mediante la estimación de un modelo Probit.

- El cálculo de los efectos marginales para cada una de las esperanzas del modelo Tobit que se describieron previamente difieren entre sí, en algunos casos, se requiere utilizar algunos resultados estadísticos importantes.
} 


\subsubsection{Efectos marginales par $E\left[y_{i} \mid x_{i}\right]$}

El efecto marginal más importante dentro del modelo Tobit, está dado por la siguiente expresión:

$$
\frac{\partial E\left[y_{i} \mid x_{i}\right]}{\partial x_{i}}=\beta \Phi\left(\frac{x_{i}^{\prime} \beta}{\sigma}\right)
$$

La descomposición de McDonald y Mofitt

En términos empíricos, quizá lo más valioso del análisis Tobit es la distinción de los efectos planteada por la siguiente relación:

$$
E\left[y_{i} \mid x_{i}\right]=\Phi\left(\frac{x_{i}^{\prime} \beta}{\sigma}\right) E\left[y_{i} \mid x_{i}, y_{i}>0\right]
$$

La cual provee una base para distinguir cuantitativamente, qué proporción del efecto global se debe a los consumidores que ya participan en el mercado, que es la derivada de la ecuación (13); y qué cantidad del efecto total obedece al cambio en la probabilidad de que entren al mercado los consumidores que actualmente no efectúan compras, la derivada de $\Phi\left(\frac{x_{i}^{\prime} \beta}{\sigma}\right)$ (Villezca y Moreno, 2000). Al seguir a McDonald y Mofitt (1980), la descomposición del efecto global para la i-ésima variable explicativa $x_{i}$, se obtiene diferenciando parcialmente $E\left[y_{i} \mid x_{i}\right]$ y se recurre al resultado de la ecuación (10). Luego, se deriva esta expresión con respecto a $x_{i}$. Por consiguiente, la derivada anterior se puede expresar como:

$\frac{\partial E\left[y_{i} \mid x_{i}\right]}{\partial x_{i}}=E\left[y_{i} \mid x_{i} y_{i}>0\right] \frac{\partial \operatorname{Pr}\left(y_{i}>0 \mid x_{i}\right)}{\partial x_{i}}+\operatorname{Pr}\left(y_{i}>0 \mid x_{i}\right) \frac{\partial E\left[y_{i} \mid x_{i}, y_{i}>0\right]}{\partial x_{i}}$

La cual se conoce como la descomposición de McDonald y Mofitt.

Cada uno de los componentes de la ecuación (34) tiene una interpretación particular.

$$
\operatorname{Pr}\left(y_{i}>0 \mid x_{i}\right)=\Phi\left(\frac{x_{i}^{\prime} \beta}{\partial}\right)
$$


El término anterior, mide la probabilidad de aquellas personas que iniciando en $y=0$ podrían escoger $y>0$ (por ejemplo, la probabilidad de que

\section{RubÉN S ANTOS-PÉREZ}

un individuo que no consume un bien, lo consuma, es decir que ingrese al mercado). A su vez, el término:

$$
E\left[y_{i} \mid x_{i}, y_{i}>0\right]=x_{i}^{\prime} \beta+\sigma \lambda_{i}
$$

Mide el valor esperado de y para la subpoblación donde y es positivo (por ejemplo, el consumo promedio para las personas que ya consumen el bien en cuestión).

En suma, el efecto global sobre $y_{i}$ de la ecuación (16), se divide en dos partes: (i) el cambio en $y_{i}$ para aquellas observaciones arriba del límite, ponderado por la probabilidad de estar por arriba del límite y; (ii) el cambio en la probabilidad de estar por arriba del límite, ponderado por el valor esperado de $y_{i}$ que está por arriba del límite. ${ }^{8}$

Expresión de $E\left[y_{i} \mid x_{i}\right.$, ]en forma de elasticidades

Para fines de interpretación económica, y para estandarizar las unidades de

medición, $E\left[y_{i} \mid x_{i}\right.$, ]se puede descomponer en forma de elasticidades:

$$
\begin{gathered}
\frac{\bar{x}}{E\left[y_{i} \mid x_{i}\right]} \frac{\partial E\left[y_{i} \mid x_{i}\right]}{\partial x_{i}}= \\
\frac{\bar{x}}{\overline{P r\left(y_{i}>o \mid x_{i}\right)}} \cdot \frac{\partial \operatorname{Pr}\left(y_{i}>0 \mid x_{i}\right.}{\partial x_{i}}+\frac{\bar{x}}{E\left[y_{i} \mid x_{i}, y_{i}>0\right]} \cdot \frac{\partial E\left[y_{i} \mid x_{i} y_{i}>0\right]}{\partial x_{i}}
\end{gathered}
$$

en donde:

* Una descomposición que es útil para calcular numéricamente el efecto de McDonald y Moffit emplea la derivada dada por:

Al simplificar un poco más se tiene:

$$
\frac{\partial\left[V_{1} \mid x_{1}\right]}{\partial x_{1}}=\frac{\partial}{\partial x_{1}}\left(x_{i} \beta+\sigma \lambda_{i}\right)+\left(x_{i} \beta+\sigma \lambda_{i}\right) \frac{\partial}{\partial x_{i}} \phi_{i}
$$

$$
\frac{\partial E\left[y_{1} \mid x_{1}\right]}{\partial x_{1}}=\Phi_{i} \times \beta\left[1-\lambda_{i}\left(\alpha_{i}+\lambda_{i}\right)\right]+\left(x_{i} \beta+\sigma \lambda_{i}\right) \phi_{i} x\left(\frac{\beta}{\sigma}\right)
$$

Esta última expresión es la descomposición de McDonald y Moffit. La diferencia entre esta última y la ecuación (16) es que la primera es la expresión desarrollada de la ecuación (16).

Otra forma alternativa de presentar la ecuación anterior puede ser:

en donde:

$$
\frac{\partial E\left[y_{1} \mid x_{1}\right]}{\partial x_{1}}=\left\{\beta \phi_{i}\left[1-\lambda_{i}\left(\alpha_{i}+\lambda_{i}\right)\right]+\phi_{i}\left(\alpha_{i}+\lambda_{i}\right)\right\}
$$

$$
\alpha_{i}=\frac{x_{i} \beta}{\sigma}, \phi_{i}=\Phi\left(\alpha_{i}\right), \phi_{i}=\Phi\left(\alpha_{i}\right) \text { y } \lambda_{i}=\frac{\phi_{i}}{\phi_{i}} .
$$




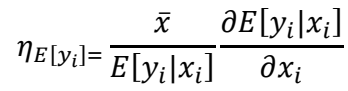

$$
\begin{aligned}
& \eta_{\Phi\left[z_{i}\right]=} \frac{\bar{x}}{\operatorname{Pr}\left(y_{i}>0 \mid x_{i}\right)} \frac{\partial \operatorname{Pr}\left(y_{i}>0 \mid x_{i}\right)}{\partial x_{i}} \\
& \eta_{E\left[y *_{i}\right]}=\frac{\bar{x}}{E\left[y_{i} \mid x_{i} y_{i}>0\right]} \frac{\partial E\left[y_{i} \mid x_{i}, y_{i}>0\right]}{\partial x_{i}}
\end{aligned}
$$

Con lo cual, se escribe de manera compacta el resultado anterior:

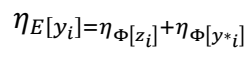

Ésta es una expresión alternativa de la descomposición de McDonald y Moffit, en términos de elasticidades.

\subsubsection{Efectos marginales para $E\left[y_{i}^{*} \mid x_{i}\right]$}

Éstos son muy fáciles de obtener, puesto que simplemente son los coeficientes $\beta$ del modelo Tobit, esto es:

$$
\frac{\partial E\left[y_{i} \mid x_{i}\right]}{\partial x_{i}}=\beta
$$

Este efecto rara vez se utiliza, debido a que $y_{i}^{*}$ no se observa.

\subsubsection{Efectos marginales para $E\left[y_{i} \mid x_{i} y_{i}>0\right]$}

Este efecto se obtiene fácilmente, dado que éste ya se ha calculado antes; dicho resultado se expresa como:

$$
\frac{E\left[y_{i} \mid x_{i} y_{i}>0\right]}{\partial x_{i}}=\beta\left[1-\lambda_{i}\left(\alpha_{i}+\lambda_{i}\right)\right]
$$

\subsection{Estimaciones del modelo Tobit}

Bajo la presencia de censuramiento de los datos, el estimador de MCO es inconsistente, por ello la estimación del modelo Tobit se realiza por medio del método de máxima verosimilitud (MV).

Al suponer que las primeras $j$ observaciones son iguales a cero y el resto distintas de cero, la función de verosimilitud sería como sigue: 


$$
L=\prod_{i=1}^{j} \operatorname{Pr}\left(Y_{i}=0\right) \prod_{i=j+1}^{n} \operatorname{Pr}\left(Y_{i}>0\right)
$$

dado que

$$
\operatorname{Pr}\left(y_{i}=0\right)=1-\Phi\left(\frac{x_{i}^{\prime} \beta}{\sigma}\right)
$$

y

$$
\operatorname{Pr}\left(Y_{i}>0\right)=\phi\left(\frac{y_{i}-x_{i}^{\prime} \beta}{\sigma}\right)=\frac{1}{\sigma \sqrt{2 \pi}} e^{-\frac{1}{2}\left(\frac{y_{i}-x_{i}^{\prime} \beta}{\sigma}\right)^{2}}
$$

al sustituir (20) y (21) en (19), se tiene el siguiente resultado:

$$
L=\prod_{j=1}^{j}\left[1-\Phi\left(\frac{x_{i}^{\prime} \beta}{\sigma}\right)\right] \prod_{i=j+1}^{n}\left[\frac{1}{\sigma \sqrt{2 \pi}} e^{-\frac{1}{2}\left(\frac{y_{i}-x_{i}^{\prime} \beta}{\sigma}\right)^{2}}\right]
$$

al tomar logaritmos en ambos lados de (22):

$$
\operatorname{In} L=\sum_{i=1}^{j} \operatorname{In}\left[1-\Phi\left(\frac{x_{i}^{\prime} \beta}{\sigma}\right)\right]-\frac{1}{2} \sum_{i=j+1}^{n}\left[\operatorname{In}(2 \pi)+\operatorname{In} \sigma^{2}+\left(\frac{y_{i}-x_{i}^{\prime} \beta}{\sigma}\right)^{2}\right]
$$

De esta manera, la estimación por MV del modelo Tobit involucra dividir las observaciones en dos conjuntos. El segundo término de (23) contiene las observaciones no censuradas, en donde el método de MV las trata(23) igual que $\mathrm{MCO}$ (note la similitud con su función de verosimilitud). El primer término contiene las observaciones censuradas, para estas observaciones, no se conoce el valor específico de $\mathrm{y}^{*}$, pero se puede proceder calculando la probabilidad de estar censurado, y se incorpora esta cantidad en la función de verosimilitud (Long, 1997).

Por último, dada la naturaleza de especial de la función de verosimilitud del modelo Tobit, los teoremas usuales acerca de los estimadores de MV no son válidos. No obstante, Amemiya (1973) demostró que la maximización de (23) con respecto a los parámetros desconocidos $\beta$ y $\sigma^{2}$ conduce a la obtención de estimadores que poseen las propiedades asintóticas de consistencia y eficiencia. 


\section{Estimación de un modelo Tobit para la demanda de transporte privado en la Zona Metropolitana del Valle de México}

En esta sección se estima un modelo Tobit de los determinantes de la demanda de uso de autos particulares por parte de los hogares de la ZMVM para el año 2012. El objetivo es identificar las variables más importantes que determinan este fenómeno; con ese propósito se emplean las herramientas analíticas que se desarrollaron en la sección anterior.

\subsection{La base de datos}

De manera análoga al trabajo de Nolan (2002), la base de datos que se emplea en este documento se construye a partir de la Encuesta Nacional de Ingresos y Gastos de los Hogares (ENIGH) para el año 2012. La muestra consta de 3136 hogares localizados en la ZMVM.

En la tabla 1 se presenta la definición de las variables que se emplearán en el estudio econométrico. Siguiendo la metodología empleada por Nolan (2002), en el presente trabajo se recurre al gasto per cápita en gasolina por hogar como proxy del uso del vehículo; no obstante, a diferencia del trabajo de este autor, en la presente investigación, se emplea el ingreso corriente total en lugar del gasto total, como medida del ingreso del hogar. En la tabla 2 se presentan las estadísticas descriptivas de las variables de la tabla 1 .

De acuerdo con esta información, el gasto total per cápita promedio en gasolina por hogar es de 133.47 pesos al mes, con una desviación estándar de 273.52 pesos; además, el ingreso per cápita promedio de los hogares que componen la muestra es de 5200.79 pesos mensuales, con una desviación estándar de 7674.09 pesos. El 38\% de los hogares destina una parte de su ingreso a la compra de gasolina, lo que equivale a decir que una fracción cercana a esa proporción de hogares cuenta con al menos un auto o vehículo de motor. 


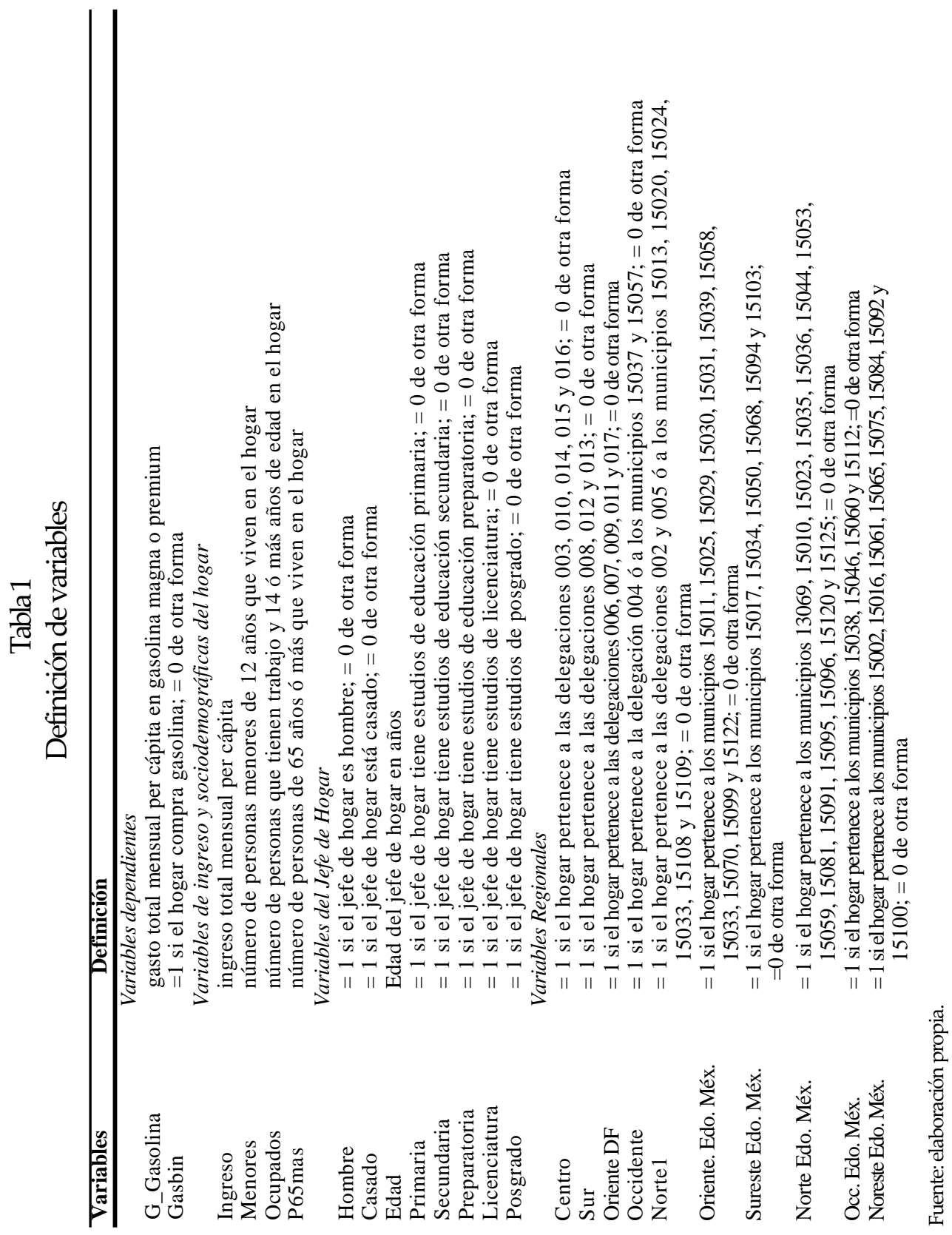


Cabe mencionar que algunas características importantes sobre los jefes de hogar de la muestra son las siguientes: su edad promedio es de 49.5 años; casi una cuarta parte de ellos está casado; $31 \%$ de los ellos cuenta con un nivel máximo de secundaria y sólo el $4 \%$ de ellos tiene posgrado.

Por último, vale precisar que cerca del $21 \%$ de los hogares se ubican en las delegaciones Cuauhtémoc, Benito Juárez, Miguel Hidalgo, Coyoacán y Álvaro Obregón (zona centro); seguidos del 19\% de las unidades familiares que se encuentran en las delegaciones Venustiano Carranza, Tláhuac, Iztacalco, Iztapalapa o Milpa Alta (zona oriente DF); y poco más de $15 \%$ de la muestra se localiza en la región norte 1 , conformada por las delegaciones Azcapotzalco, Gustavo A. Madero y por los municipios de Tlalnepantla, Ecatepec, Coacalco, Tultitlán, Atizapán, Cuautitlán y Tultepec, pertenecientes al Estado de México, siendo estas tres, las regiones más representativas de la muestra. 
Tabla 2

Estadísticas descriptivas de la base de datos

\begin{tabular}{|c|c|c|c|c|c|}
\hline Variable & Observaciones & Media & $\begin{array}{c}\text { Desviación } \\
\text { estándar }\end{array}$ & Mínimo & Máximo \\
\hline \multicolumn{6}{|c|}{ Variables dependientes } \\
\hline G_Gasolina & 3136 & 133.47 & 273.52 & 0.00 & 3000.00 \\
\hline Gasbin & 3136 & 0.381 & 0.486 & 0 & 1 \\
\hline \multicolumn{6}{|c|}{ Variables de ingreso y sociodemográficas del hogar } \\
\hline Ingreso & 3136 & 5200.79 & 7674.09 & 0.00 & 90983.60 \\
\hline Menores & 3136 & 0.8 & 1.0 & 0 & 5 \\
\hline Ocupados & 3136 & 1.9 & 1.1 & 0 & 5 \\
\hline P65mas & 3136 & 0.3 & 0.5 & 0 & 3 \\
\hline \multicolumn{6}{|c|}{ Variables del jefe de hogar } \\
\hline Hombre & 3136 & 0.747 & 0.435 & 0 & 1 \\
\hline Casado & 3136 & 0.240 & 0.427 & 0 & 1 \\
\hline Edad & 3136 & 49.5 & 14.8 & 17 & 94 \\
\hline Primaria & 3136 & 0.212 & 0.409 & 0 & 1 \\
\hline Secundaria & 3136 & 0.310 & 0.463 & 0 & 1 \\
\hline Preparatoria & 3136 & 0.159 & 0.366 & 0 & 1 \\
\hline Licenciatura & 3136 & 0.166 & 0.372 & 0 & 1 \\
\hline Posgrado & 3136 & 0.040 & 0.196 & 0 & 1 \\
\hline \multicolumn{6}{|c|}{ Variables regionales } \\
\hline Centro & 3136 & 0.206 & 0.404 & 0 & 1 \\
\hline Sur & 3136 & 0.094 & 0.292 & 0 & 1 \\
\hline Oriente DF & 3136 & 0.193 & 0.395 & 0 & 1 \\
\hline Occidente & 3136 & 0.031 & 0.172 & 0 & 1 \\
\hline Norte1 & 3136 & 0.154 & 0.361 & 0 & 1 \\
\hline Oriente Edo. Méx. & 3136 & 0.111 & 0.314 & 0 & 1 \\
\hline Sureste Edo. Méx. & 3136 & 0.000 & 0.000 & 0 & 0 \\
\hline Norte Edo.Méx. & 3136 & 0.054 & 0.226 & 0 & 1 \\
\hline Occ. Edo. Méx. & 3136 & 0.044 & 0.204 & 0 & 1 \\
\hline Noreste Edo. Méx. & 3136 & 0.025 & 0.155 & 0 & 1 \\
\hline
\end{tabular}

Fuente: elaboración propia. 


\subsection{Estimaciones y resultados}

Para analizar el gasto en gasolina efectuado por los hogares, se emplean funciones de Engel, ${ }^{9}$ que para el caso específico del presente estudio se considera la siguiente forma funcional:

$$
P Q=Y_{o}+\sum_{i=1}^{k} Y_{j} X_{j}+\sum_{i=1}^{m} \delta_{j} Z_{j}+\mu
$$

En donde $P Q$ es el gasto per cápita en gasolina para cada uno de los hogares; $X_{j}$ son los términos lineales para las variables explicativas; $Z_{j}$ son los términos cuadráticos; $u$ es un término de error; $\mathrm{y} \gamma_{0}, \gamma_{j} \mathrm{y} \delta_{j}$ son parámetros que tienen que ser estimados. La función incorpora factores como características del jefe de hogar, composición de la familia, características de los integrantes del hogar, región, entre otras.

El modelo anterior se estima por el método de mínimos cuadrados ordinarios (MCO), así como por máxima verosimilitud (MV), a través de un modelo Tobit. Al emplear dicha especificación, se obtienen los resultados de la tabla 3.

A partir de los resultados de la tabla 3, destaca el hecho que los coeficientes que arrojan ambos modelos son relativamente similares, incluso su significancia estadística, además de que ambos modelos presentan un buen ajuste. No obstante, según Wooldridge (2010), aunque es tentador comparar los coeficientes de los dos modelos esto no es muy informativo, ya que a diferencia de los coeficientes del modelo de regresión lineal, los del modelo Tobit no tienen una interpretación directa.

En la figura 1 se muestran los valores estimados mediante el modelo To-bit y los valores observados para el gasto en gasolina per cápita por hogar. ${ }^{10} \mathrm{~A}$ su vez, en la figura 2 se muestran los valores estimados por MCO. La diferencia principal entre las dos estimaciones radica en que el modelo lineal arroja valores predichos negativos, mientras que el modelo Tobit por construcción,

Las funciones de Engel parten del suptiesto que todos los precios se mantienen constantes, lo que se adecua bastante bien a los datos de corte transversal, ya que éstos se recopilan en un punto del tiempo, de tal manera que no se presenta variabilidad en los precios.

${ }^{10}$ Los valores predichos por el modelo Tobit se generan mediante la fórmula:

$$
\widehat{y}_{\iota}=\Phi\left(\frac{x_{i}^{\prime} \beta}{\hat{\sigma}}\right) x_{i}^{\prime} \beta+\delta \phi\left(\frac{x_{i}^{\prime} \beta}{\hat{\sigma}}\right)
$$


siempre da como resultado valores predichos mayores o iguales a cero. En la figura 2 se observa que la ecuación de predicción del modelo Tobit no es lineal, ya que para una buena parte de los valores que son cero, el modelo arroja valores predichos bastante cercanos a esas observaciones; posteriormente, presenta un punto de quiebre hacia arriba, a partir de lo cual se ajusta a los valores observados que son positivos.

Tabla 3

Estimaciones por MCO y Tobit para la demanda de gasolina

\begin{tabular}{|lcc|}
\hline \multicolumn{1}{c}{ Variable } & MCO Tobit & \\
\hline Ingreso & 0.0187 & 0.0332 \\
Ingreso 2 & $(17.89)^{* * *}$ & $(15.53)^{* * *}$ \\
& $6.72 \mathrm{E}-08$ & $-9.11 \mathrm{E}-08$ \\
Menores & $(4.42)^{* * *}$ & $(-3.02)^{* * *}$ \\
& 2.2392 & 9.6062 \\
Ocupados & $(0.60)^{\prime}$ & $(1.04)^{\prime}$ \\
& -7.7361 & 15.7106 \\
P65mas & $(-2.41)^{* *}$ & $(2.09)^{* *}$ \\
& -31.5468 & -88.8542 \\
Hombre & $(-4.11)^{* * * \prime}$ & $(-4.81)^{* * *}$ \\
& 30.8096 & 85.9327 \\
Casado & $(3.77)^{* * *}$ & $(4.37)^{* * *}$ \\
& 23.4981 & 79.4628 \\
Edad & $(2.91)^{* * *}$ & $(4.43)^{* * *}$ \\
& 0.6323 & 4.5500 \\
Primaria & $(2.03)^{* * \prime}$ & $(6.21)^{* * *}$ \\
& 26.4081 & 162.8580 \\
Secundaria & $(2.27)^{* *}$ & $(5.30)^{* * *}$ \\
& 7.5065 & 160.7817 \\
Preparatoria & $(0.63)^{\prime}$ & $(5.23)^{* * *}$ \\
Licenciatura & 82.7114 & 331.8274 \\
& $(6.00)^{* * *}$ & $(9.66)^{* * *}$ \\
& 157.3666 & 413.1036 \\
\hline
\end{tabular}


Tabla 3. Conclusión.

\begin{tabular}{|lll|}
\hline \multicolumn{1}{|c}{ Variable } & \multicolumn{1}{c|}{ MCO } & Tobit \\
\hline \multirow{2}{*}{ Posgrado } & $(10.11)^{* * *}$ & $(11.34)^{* * *}$ \\
& -74.0091 & -78.8707 \\
Sur & $(-3.84)^{* * *}$ & $(-2.09)^{* *}$ \\
& -18.8729 & -36.0787 \\
Oriente DF & $(-1.49)^{\prime}$ & $(-1.21)^{\prime}$ \\
& 9.0600 & 63.8488 \\
Occidente & $(0.93)^{\prime}$ & $(2.96)^{* * *}$ \\
& 74.0012 & 107.1175 \\
Norte 1 & $(3.71)^{* * *}$ & $(2.30)^{* *}$ \\
& -38.1995 & -70.8046 \\
Oriente. Edo. Méx. & $(-3.62)^{* * *}$ & $(-2.92)^{* * *}$ \\
& -47.0991 & -192.0787 \\
Norte Edo. Méx. & $(-3.92)^{* * *}$ & $(-6.05)^{* * *}$ \\
& -31.8084 & -18.6267 \\
Occ. Edo. Méx. & $(-1.97)^{* * \prime}$ & $(-0.47)^{\prime}$ \\
& -5.7771 & 7.8968 \\
Noreste Edo. Méx. & $(-0.32)^{\prime}$ & $(0.18)^{\prime}$ \\
Constante & -26.6296 & -39.9980 \\
R2 & $(-1.19)^{\prime}$ & $(-0.72)^{\prime}$ \\
Sigma & -41.3777 & -780.2214 \\
F & $(-1.77)^{* \prime}$ & $(-13.12)^{* * *}$ \\
& 0.5551 & 0.5436 \\
& $183.0500^{\prime}$ & 345.6127 \\
\hline
\end{tabular}

Fuente: elaboración propia.

$\left(^{*}\right)\left({ }^{* *}\right)\left(^{* * *}\right)$ Coeficientes significativos al $10 \%, 5 \%$ y $1 \%$, respectivamente. 


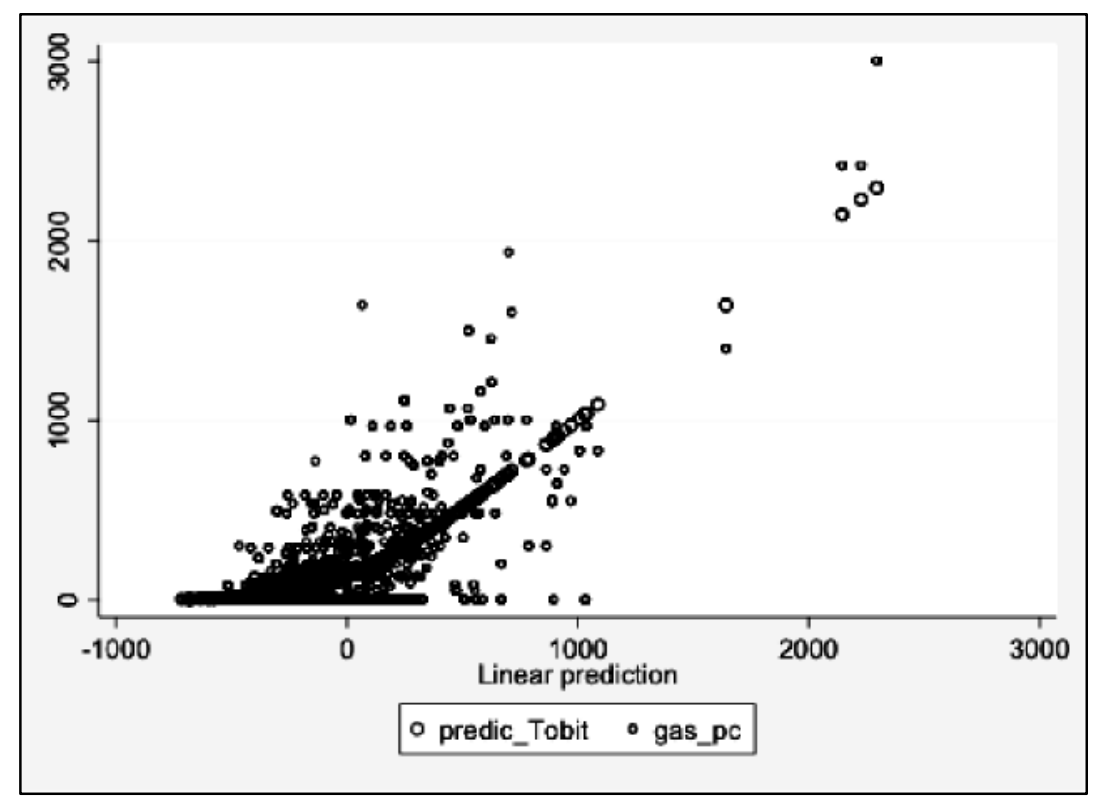

Fuente: elaboración propia.

Figura 1

Valores observados y estimados por el modelo Tobit para el gasto en gasolina por hogar per cápita.

Debido a que los coeficientes del modelo Tobit no tienen una interpretación directa, en la tabla 4, se calculan los efectos marginales para este modelo, así como la descomposición de McDonald y Moffit para cada uno de los coeficientes del mismo.

Con base en los resultados de las tablas 3 y 4 , se observa que la mayor parte de las variables del modelo Tobit son estadísticamente significativas; aunque no sucede lo mismo con algunas variables regionales y con la variable menores. 


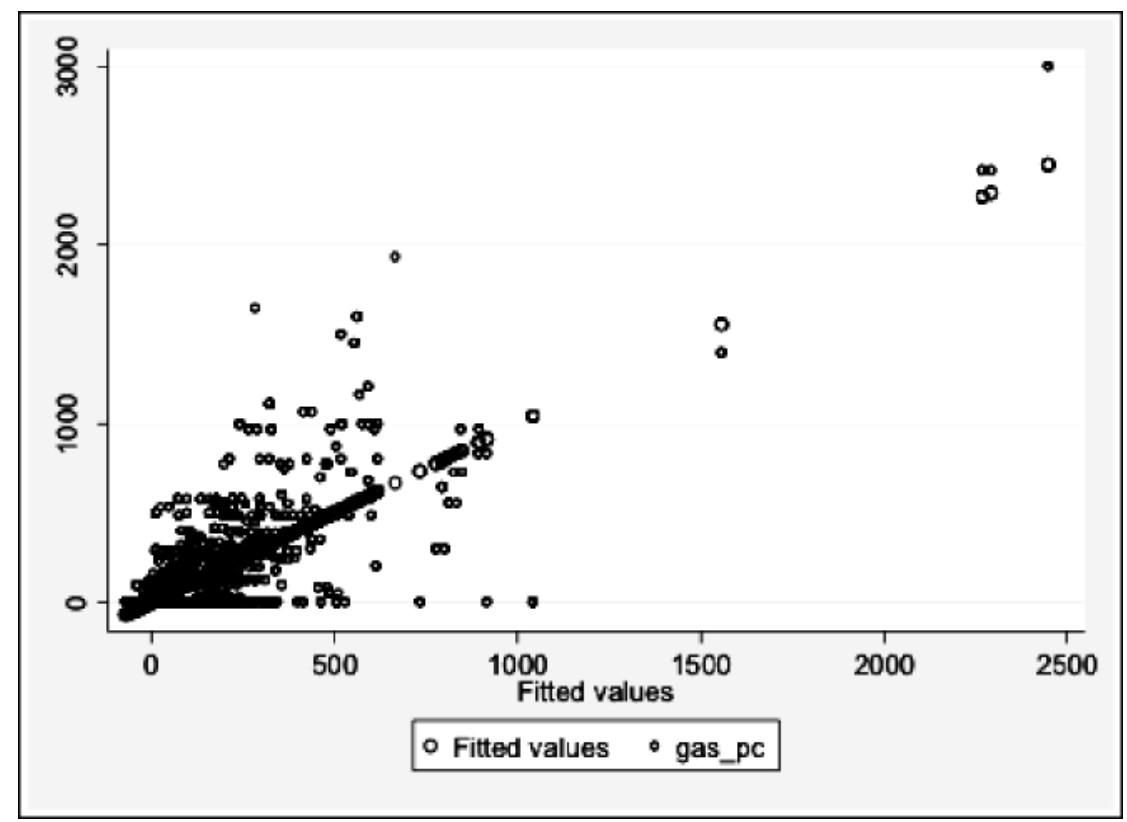

Fuente: elaboración propia.

Figura 2

Valores observados y estimados por el modelo lineal para el gasto en gasolina por hogar per cápita

Específicamente, las variables ocupados y p65mas tienen una significan-cia estadística en la explicación del gasto en gasolina (variable proxy del uso del auto); el efecto de la primera puede interpretarse de la siguiente manera: por cada persona ocupada adicional en el hogar, el gasto en gasolina aumentará casi seis pesos en términos per cápita; ello como un resultado de una mayor competencia por el vehículo o vehículos dentro de los hogares. Esto también tiene que ver con el hecho de que estas personas tienen mayores posibilidades de adquirir un auto. 
Tabla 4

Efectos marginales y descomposición de McDonald y Moffit del modelo Tobit para la demanda de gasolina

\begin{tabular}{|c|c|c|c|c|c|}
\hline \multirow{2}{*}{ Coeficiente } & $\partial E\left[y_{i} \mid x_{i}, y_{i}>0\right]$ & $\partial E\left[y_{i} \mid x_{i}\right]$ & $\partial \operatorname{Pr}\left(y_{i}>0 \mid x_{i}\right)$ & $\operatorname{Pr}\left(y_{i}>0 \mid x_{i}\right) \partial E\left[y_{i} \mid x_{i}, y_{i}>0\right]$ & $E\left[y_{i} \mid x_{1} y_{i}>0\right] \quad \partial \operatorname{Pr}\left(y_{i}>0 \mid x_{i}\right)$ \\
\hline & $\partial x_{i}$ & $\partial x_{i}$ & $\partial x_{i}$ & $\partial x_{i}$ & $\partial x_{i}$ \\
\hline Ingreso & 0.0125 & 0.0000 & 0.0109 & 0.0069 & 0.0056 \\
\hline Ingreso 2 & $-3.43 \mathrm{E}-08$ & $-7.59 \mathrm{E}-11$ & $-2.98 \mathrm{E}-08$ & $-1.90 \mathrm{E}-08$ & $-1.53 \mathrm{E}-08$ \\
\hline Menores & 3.6130 & 0.0080 & 3.1408 & 2.0026 & 1.6105 \\
\hline Ocupados & 5.9090 & 0.0131 & 5.1367 & 3.2751 & 2.6339 \\
\hline P65mas & -33.4193 & -0.0740 & -29.0515 & -18.5231 & -14.8962 \\
\hline Hombre & 32.3205 & 0.0716 & 28.0963 & 17.9141 & 14.4064 \\
\hline Casado & 29.8871 & 0.0662 & 25.9809 & 16.5653 & 13.3218 \\
\hline Edad & 1.7113 & 0.0038 & 1.4876 & 0.9485 & 0.7628 \\
\hline Primaria & 61.2532 & 0.1357 & 53.2475 & 33.9504 & 27.3028 \\
\hline Secundaria & 60.4723 & 0.1340 & 52.5687 & 33.5176 & 26.9547 \\
\hline Preparatoria & 124.8050 & 0.2765 & 108.4932 & 69.1748 & 55.6302 \\
\hline Licenciatura & 155.3741 & 0.3442 & 135.0670 & 86.1182 & 69.2560 \\
\hline Posgrado & -29.6644 & -0.0657 & -25.7873 & -16.4419 & -13.2225 \\
\hline Sur & -13.5697 & -0.0301 & -11.7962 & -7.5212 & -6.0485 \\
\hline Oriente DF & 24.0144 & 0.0532 & 20.8758 & 13.3103 & 10.7041 \\
\hline Occidente & 40.2884 & 0.0892 & 35.0228 & 22.3304 & 17.9580 \\
\hline Norte 1 & -26.6306 & -0.0590 & -23.1500 & -14.7604 & -11.8703 \\
\hline Oriente Edo. Méx. & -72.2436 & -0.1600 & -62.8014 & -40.0419 & -32.2016 \\
\hline Norte Edo. Méx. & -7.0058 & -0.0155 & -6.0901 & -3.8830 & -3.1227 \\
\hline Occ. Edo. Méx. & 2.9701 & 0.0066 & 2.5819 & 1.6462 & 1.3239 \\
\hline Noreste Edo. Méx. & -15.0438 & -0.0333 & -13.0776 & -8.3382 & -6.7056 \\
\hline
\end{tabular}

Fuente: elaboración propia.

En relación a las características que tiene el jefe de hogar, se puede concluir que el género, la edad y el nivel de escolaridad, influyen de manera significativa en el uso del vehículo particular por parte de los hogares de la ZMVM. Los hogares encabezados por hombres gastan, en promedio 32 pesos per cápita más en gasolina que los hogares encabezados por mujeres. En este sentido, diversos estudios señalan que el hombre utiliza más el vehículo; Nolan (2002), por ejemplo, menciona que las mujeres, al dedicar una mayor parte de su tiempo al hogar o a realizar trabajos de medio tiempo, que requieren un menor desplazamiento, presentan una mayor disposición a caminar o a tomar el transporte público que los hombres. 
Por otro lado, es claro que a medida que aumenta el nivel de escolaridad del jefe de hogar, sus nivel de ingresos sube; con ello, éste empieza a realizar más actividades sociales, familiares, de esparcimiento o de otro tipo; razón por la cual, los hogares donde los jefes de hogar cuentan con licenciatura, gastan en promedio 155.37 pesos más que aquellos con jefes de hogar que no terminaron la primaria o que no cuentan con algún nivel de escolaridad (categoría base).

En sí, el ingreso es una de las variables más importantes que explica el uso del automóvil y que refleja los costos en los que se incurre al utilizarlo, como son: la gasolina, la tenencia, las verificaciones, los seguros, entre otros; pese a los cuales, los hogares con mayores ingresos valoran más la comodidad de conducir su vehículo, en relación a otras alternativas, como puede ser utilizar el transporte público. Además, las personas con ingresos mayores le asignan un mayor valor a los ahorros de tiempo.

En este sentido, retomando los resultados de la tabla 4, destaca el hecho que ante un aumento del ingreso de 100 pesos per cápita al mes, el gasto por persona en gasolina aumentará en promedio 1.25 pesos en el mismo periodo.

Acorde con lo anterior, el Programa para Mejorar la Calidad del Aire de la ZMVM 2002-2010 establece que el crecimiento económico da lugar a una mayor congestión vial y contaminación ambiental, al impactar directamente a las tasas de motorización, la generación de viajes y el crecimiento urbano. Asimismo, el mayor ingreso incrementa la posesión de vehículos.

Dado que la ZMVM es una de las de mayor dinamismo económico en el país, la tendencia señala que en los próximos años el parque vehicular se incrementará de manera significativa, complicando aún más el problema de movilidad; por esta razón se vuelve urgente poner en marcha políticas enfocadas a la sustitución del vehículo o reducción del uso del mismo.

En la tabla 5 se expresa el efecto anterior en términos de elasticidades, ahí se observa que ante un aumento de $1 \%$ del ingreso, el gasto en gasolina sube $0.54 \%$; esa variación se debe principalmente al número de hogares que deciden ingresar al mercado, lo cual equivale a $0.38 \%$. Acorde con la magnitud de la elasticidad del ingreso, se puede apreciar que para los hogares que cuentan con vehículo, el gasto en gasolina lo consideran un bien necesario.

Además, en la tabla 5 se presentan las elasticidades para la variable menores y el resto de las variables que no son binarias. Los resultados muestran que en la mayor parte de los casos, el efecto de esas variables sobre el gasto en gasolina, se debe principalmente a los hogares que entran al mercado. 
En particular, ante un aumento de $100 \%$ de las personas ocupadas en los hogares (aumento del empleo), el gasto en gasolina por parte de éstos en términos per cápita, aumentará en $9 \%$; seis puntos porcentuales de dicho incremento se originarán por los nuevos consumidores que ingresen al mercado (y que por lo tanto adquieran autos); a su vez, tres puntos porcentuales se deberán al incremento en el consumo de los hogares que ya se hallaban en el mercado (los que ya contaban con al menos un auto).

\section{Tabla 5}

Elasticidades del modelo Tobit para la demanda de uso de gasolina

\begin{tabular}{|lrlr|}
\multicolumn{1}{c}{ Variables } & \multicolumn{1}{c}{$\eta_{E\left[y_{i}\right]}$} & \multicolumn{1}{c}{$\eta_{\Phi\left[z_{i}\right]}$} & $\eta_{E\left[y_{i}^{*}\right]}$ \\
\hline Ingreso & 0.5443 & 0.3830 & 0.1613 \\
Ingreso 2 & -0.0246 & -0.0173 & -0.0073 \\
Menores & 0.0244 & 0.0172 & 0.0072 \\
Ocupados & 0.0942 & 0.0663 & 0.0279 \\
P65mas & -0.0710 & -0.0500 & -0.0210 \\
Edad & 0.7085 & 0.4986 & 0.2099 \\
\hline
\end{tabular}

Fuente: elaboración propia.

Partiendo de estos resultados se puede apreciar que la mayoría de ellos son bastante similares a los alcanzados por Benett (1967) y Nolan (2002) en sus trabajos. De manera análoga a lo obtenido por Nolan (2002), el presente estudio demuestra que la gasolina es un bien necesario, con una elasticidad cercana al resultado obtenido por dicho autor: 0.514 , siendo el nuestro equivalente a 0.544 . No obstante, la variable menores no es significativa en nuestro trabajo, lo que marca una diferencia importante respecto al de Nolan (2002).

Por otro lado, a diferencia del trabajo de Benett (1967), el nuestro no permite corroborar de manera concluyente que el gasto destinado a gasolina sube con la edad del jefe de hogar y que ésta comienza a contraerse en la medida que el jefe de hogar se aproxima a su edad de jubilación, aunque la mayoría de los resultados de este autor se mantienen.

Cabe señalar, que aunque nuestro modelo no permite probar de manera contundente lo primero, de manera indirecta, puede apreciarse que el uso 
del vehículo aumenta con la edad del jefe de hogar y que además, la variable p65mas tiene un coeficiente negativo, lo que en cierta forma permite vislumbrar que los hogares con personas cercanas a su edad de jubilación o jubiladas, utilizarán menos el vehículo.

Por último, los estudios de Benett (1967) y Nolan (2002) subrayan que la distancia es un elemento fundamental en el uso del automóvil, por esa razón, las variables regionales tratan de tomar en cuenta esos factores. Villarreal (2009), respalda lo anterior y menciona que en el centro de la ZMVM se han asentado las oficinas corporativas de las grandes empresas nacionales y extranjeras, mientras que la producción se lleva a cabo en las fábricas que se localizan en la parte exterior de la misma; por esa razón, gran parte de los habitantes que se encuentran fuera del centro buscan alternativas de empleo en dicho lugar. Este aspecto, se retoma en la definición de la variable Centro, que se considera como la categoría base de las variables dummy regionales o de distancia.

En este tenor, los integrantes de los hogares que se localizan en la región Oriente DF, gastan en promedio 24 pesos per cápita más que los que se localizan en la región Centro, básicamente debido a que éstos tienen que realizar un mayor desplazamiento hacia sus lugares de trabajo. Algo similar a lo que ocurre para los hogares que se localizan en la región Occidente.

Por otro lado, en el caso de los hogares del Estado de México localizados a una mayor distancia del Centro, como los de las regiones Norte Edo. Méx., y Oriente Edo. Méx., que incluyen los pertenecientes a los municipios de Tecámac, Nezahualcóyotl, Chalco, Ixtapaluca, Chimalhuacán y otros que tienen gran población, hay un porcentaje relativamente pequeño de habitantes que recorren grandes distancias en automóvil privado; ya sea porque la mayor parte de los trabajadores utiliza el transporte público, o porque realizan sus labores en sus mismos municipios; pese a esto el volumen de tráfico que se genera en las vialidades por las que transitan diariamente estos contingentes es enorme. Particularmente, los hogares que habitan en la región Oriente Edo. Méx., gastan 72.24 pesos per cápita menos en gasolina en promedio, en comparación con los hogares de la región Centro.

Por último, cabe agregar que con base en el modelo estimado, se puede estimar la demanda total de vehículos (adquisición de vehículos nuevos y usados), así como su demanda en su uso diario (medida por la demanda de gasolina) dentro de la ZMVM; a partir de lo cual, se podrían realizar proyecciones sobre el volumen de unidades que conformarán el parque vehicular de la ZMVM 
en los próximos años, todo ello con la finalidad de que los gobiernos puedan realizar una adecuada planeación en la construcción de infraestructuras viales, así como para poder implementar planes de mediano y largo plazo enfocados a mejorar la movilidad de toda la ZMVM. No obstante, debido a los propósitos del presente estudio, dicho análisis se deja para futuras investigaciones.

\section{Conclusiones}

Empleando un modelo Tobit, en el presente documento se estimaron los determinantes de la demanda de uso de autos particulares en la ZMVM para el año 2012. La característica principal de dicho modelo radica que éste toma en cuenta la presencia del censuramiento de la variable dependiente en el análisis de regresión, convirtiéndose así en una alternativa para el tratamiento de variables normales censuradas en cero, resolviendo el problema de sesgo, inconsistencia e ineficiencia, que caracterizan a los modelos de regresión convencionales, ya que toma en cuenta la diferencia cualitativa entre las observaciones censuradas y las observaciones no censuradas.

Además, el análisis se enriquece con la descomposición de McDonald y Moffit, la cual permite descomponer los cambios en el consumo de un bien, en dos partes: una que considera las variaciones en la estructura de consumo para los consumidores originales y otra que toma en cuenta la probabilidad de ingresar al mercado a consumir.

De esta manera, la aplicación del análisis Tobit para estimar los determinantes de la demanda de uso de autos particulares en la ZMVM, empleando el gasto en gasolina como proxy de ésta y utilizando los datos de la INEGI 2012, entre otras cosas, permitió concluir que el ingreso es la variable más importante en la determinación de la demanda de uso del automóvil entre los hogares de la ZMVM. Además, el análisis arrojó que cerca del 40\% de los hogares destinan una parte de su ingreso a la compra de gasolina.

Al emplear la descomposición de McDonald y Moffitt, se observó que el efecto más importante del ingreso sobre el uso del vehículo se debe a los nuevos consumidores que ingresan al mercado; por lo cual, se espera que el crecimiento económico, acompañado de un aumento en el ingreso de los hogares incida en una mayor adquisición de vehículos por parte de los nuevos consumidores, lo cual con bastante seguridad, en los próximos años complicará aún más el problema de movilidad en la ZMVM. 
En el presente estudio se alcanzan resultados muy similares a los de Benett (1967) y Nolan (2002), al comprobar que las variables edad, número de personas ocupadas y distancia, afectan significativamente a la demanda de uso de autos.

Al igual que Nolan (2002), nuestro trabajo demuestra que la gasolina se comporta como un bien necesario, en donde la elasticidad es bastante cercana al resultado obtenido por este autor, a saber, 0.514 contra 0.544 obtenida por nuestro trabajo. Sin embargo, en nuestro trabajo la variable "menores" resulta ser no significativa en la demanda del uso de vehículos por parte de las familias, lo que marca una diferencia con el estudio de Nolan (2002). Asimismo, nuestros resultados no permiten comprobar de manera contundente el resultado de Benett (1967), respecto a que el gasto en gasolina aumenta con la edad y que éste empieza a disminuir a medida que el jefe de hogar se aproxima a su edad de jubilación.

Dicho lo anterior, sólo resta sugerir algunas recomendaciones de política pública con la finalidad de que la movilidad mejore en la ZMVM. En primera instancia, debido a que el ingreso es la variable más importante en el uso del vehículo, consideramos que un aumento en los impuestos al uso del automóvil particular se reflejaría en una reducción del mismo, y por lo tanto serviría para controlar la demanda del uso de los vehículos particulares, moderando así los problemas de congestión vehicular. Dichos impuestos podrían ser similares a los que se aplican actualmente, en particular, mediante el programa hoy no circula; sin embargo, creemos que la implementación de este tipo de programas sólo serían medidas inequitativas, si no se complementan con un programa completo de movilidad que contemple la modernización y reorganización del transporte público; la aplicación estricta de los reglamentos de tránsito; la construcción de infraestructuras que prioricen al peatón (construcción de corredores en avenidas principales y recuperación de calles), entre otras.

Debido a lo anterior, debe fomentarse un mayor uso de medios de transporte alternativos como la bicicleta y los vehículos ecológicos. De acuerdo con los resultados obtenidos en este trabajo, las medidas implementadas en el transporte público, como es la asignación de vagones especiales para mujeres y niños, en el caso del metro, o de secciones determinadas, en el caso del metrobus, son medidas que alientan y contribuyen al uso del transporte público por parte de este segmento de la población, ya que como lo muestran nuestros resultados, dichos grupos presentan una mayor disposición al uso del mismo. 
Al mismo tiempo, se debe de fomentar aún más el uso de vehículos ecológicos, como es el caso de la bicicleta, por parte de los segmentos de población de mayores ingresos y de mayor preparación educativa, quienes desarrollan mayores actividades sociales, de trabajo y familiares y que por ello tienden a usar en mayor medida el vehículo (según nuestras estimaciones), ya que ello permitiría descargar de manera importante el volumen de tráfico que se genera en sus zonas de influencia.

No obstante, para ello resulta necesario reforzar la aplicación de los reglamentos de tránsito, con un enfoque en la protección de la integridad de los conductores de dichos vehículos, al mismo tiempo que buscar el ordenamiento de la circulación de los mismos. Asimismo, se debe ampliar la infraestructura destinada a este tipo de vehículos, de esta manera, se estarían creando las condiciones necesarias para que una mayor parte de la población tenga mayores incentivos para usar la bicicleta. Adicionalmente se deben realizar mayores campañas de publicidad destinadas a usar en mayor medida el uso de este tipo de vehículos, así como enfocadas en una mayor promoción de la cultura vial.

Por último, se debe recalcar que las medidas puestas en práctica poco pueden contribuir a mejorar la movilidad de la ZMVM, sino existe una amplia coordinación entre las autoridades del Distrito Federal y las del Estado de México, porque mientras el Gobierno del Distrito Federal realice grandes esfuerzos para mejorar la movilidad dentro de su jurisdicción, éstos sólo tendrán resultados limitados si las autoridades mexiquenses no actúan de manera coordinada con las primeras. 


\section{Referencias}

Amemiya, T. (1973). “Regression analysis when the dependent variable is truncated normal", Econometrica, 41, pp. 997-1016. (1984). "Tobit Models: A survey", Journal of Econometrics, 24, pp. 3-63.

Benett, W. (1967). "Cross-Section studies of the consumption of automóviles in the United States, American Economic Review, vol. 57 (4), pp. 841-850.

Ben-Akiva, M. y S. Lerman (1975). “Disaggregate behavioural model of automovile ownership", Transportation Research Record, 569, pp. 34-51.

Cabrer, B.; A. Sancho y G. Serrano (2001). Microeconometría y decisión, Ediciones Pirámide, Madrid, pp. 72-95.

De Donnea, F. (1971). The determinants of transport mode choice in dutch cities: some disaggregate stochastic choice models, Rotterdam, Rotterdam University Press.

Del Muro Guerrero, José M. (2006). Los determinantes de las decisiones de transporte de los hogares de México: un estudio microeconométrico. Tesis de maestría, El Colegio de México.

De Palma, A. y D. Rochat (2000). “Mode choices for trips to work in Geneva: An empirical analysis", Journal of Transport Geography, 8, 43-51.

De Souza (1999). El uso del transporte por los hogares y la contaminación atmosférica en las ciudades: un análisis comparativo entre Tailandia, México y los Estados Unidos, Population Reference Bureau, Washington D.C., pp. 28-55.

García Robles, Carmina (2010). Escenarios de consumo de energía y emisiones de gases de efecto invernadero del transporte de pasajeros de la Zona Metropolitana de la Ciudad de México, Tesis de maestría, Universidad Nacional Autónoma de México, pp.66-92.

Gobierno del Distrito Federal (2013). Inventario de emisiones contaminantes y de efecto invernadero 2012, Secretaría del Medio Ambiente, México, pp. 120-170.

Goldberger, Arthur (1964). Econometric Theory, John Wiley and Sons, New York, pp. 80-112.

Greene, W. H. (2012). Econometric Analysis, 7th ed., Prentice Hall, New Jersey, pp. 4063. Instituto de Políticas para el Transporte y el Desarrollo México (2014). Menos cajones, más ciudad. El estacionamiento de la Ciudad de México, México, pp. 240-281. (2012a). Guía de estrategias para la reducción del uso del auto en ciudades Mexicanas, México, pp. 27-52.

(2012b). La importancia de reducción del uso del automóvil en México, México, pp. 115-170.

Instituto Mexicano para la Competitividad. "Movilidad competitiva en la Zona Metropolitana de la Ciudad de México: diagnóstico y soluciones factibles", 
consultado el 16 de septiembre de 2014, disponible en http://imco.org.mx/wpcon-tent/uploads/2012/1/costos congestion en zmvm2 final abril.pdf.

Islas, V. (2000). Llegando tarde al compromiso: la crisis del transporte en la Ciudad de México, México, DF, El Colegio de México, pp. 75-98.

Long, J. Scott (1997). Regression Models for Categorical and Limited Dependent Variables, SAGE Publications, Inc., pp.190-215.

Márquez, López Lisett y Emilio Pradilla Cobos (2007). “Ciudad de México: el automóvil contra el transporte público", Investigación y Diseño, núm. 4, 2007, División de Ciencias y Artes para el Diseño, Universidad Autónoma Metropolitana, Xochimilco, México DF, México, pp. 247-285.

McDonald, J. F. y R. A. Moffit (1980). "The uses of Tobit Analysis", The Review of Economics and Statistics, 62, pp. 318-321.

Ming-Chu, P. (1994). Household travel consumption: Applications of Limited Dependent Variable models, Ph.D. Thesis, Clemson University, pp. 79-99.

Nolan, A. (2002). "The determinants of urban households' transport decisions: A microeconometric study using Irish data", Working Paper.

Smith, J. (2004). “Censored Regression Lectures”, Lecture Notes, pp. 82-110.

Thobani, M. (1984). "A Nested Logit Model of travel mode to work and auto owner-ship", Journal of Urban Economics, 15, pp. 287-301.

Tobin, J. (1958). "Estimation of Relationships for Limited Dependent Variables", Econometrica, 26, pp. 24-36.

Train, K. (1980). "A Structured Logit Model of auto ownership and mode choice", Review of Economic Studies, 28, pp. 357-370.

Villarreal G., Diana R. (2009), “Sistema de transporte y desplazamientos al trabajo en la Zona Metropolitana del Valle de México 1994-2007", Revista Transporte y Territorio, núm. 1, Universidad de Buenos Aires, pp. 130-160.

Villezca, Becerra Pedro A. y Jorge O. Moreno Treviño (2000). “Análisis del consumo de cerveza en el área Metropolitana de Monterrey: un modelo de respuesta censurada", Estudios Económicos, 15 (6), pp. 249-280.

Wooldridge, J. M. (2010). Introducción a la econometría, Cengage Learning Editores, 4⿳亠丷⿵冂. ed., México, pp. 95-120.

Apéndices

En esta parte se deriva la función generadora de momentos de una variable aleatoria normal truncada a la izquierda de $\mathrm{y}=\mathrm{a}$. Con ese propósito, se parte de la 
definición de una función generadora de momentos de una variable $(A .1)$ aleatoria y, la cual se denotada como $m_{Y}(\mathrm{t})$ y que está dada por:

$$
m_{Y}(t)=E\left[e^{t Y}\right]=\int_{-\infty}^{\infty} e^{t Y} f(y) d y
$$

En el caso particular de una variable aleatoria normal con media $\mu$ y varianza $\sigma^{2}$, su función generadora de momentos es la siguiente:

$$
m_{y}(t)=e^{\mu t+\frac{1}{2} \sigma^{2} t^{2}}
$$

Al sustituir la función de densidad de una variable aleatoria normal normal y, con media $\mu$ y varianza $\sigma^{2}$, se tiene que:

$$
m_{Y}(t)=E\left[e^{t Y}\right]=\int_{-\infty}^{\infty} e^{t Y} \frac{1}{\sigma \sqrt{2 \pi}} e^{\frac{1}{2}\left(\frac{y-\mu}{\sigma}\right)^{2} d_{y}}
$$

luego, se divide la integral anterior en dos partes:

$$
m_{Y}(t)=\int_{-\infty}^{\infty} e^{t Y} \frac{1}{\sigma \sqrt{2 \pi}} e^{\frac{1}{2}\left(\frac{y-\mu}{\sigma}\right)^{2} d_{y}}+\int_{-\infty}^{\infty} e^{t Y} \frac{1}{\sigma \sqrt{2 \pi}} e^{\frac{1}{2}\left(\frac{y-\mu}{\sigma}\right)^{2} d_{y}}
$$

al multiplicar y dividir la segunda integral por 1-F(a), se obtiene lo siguiente:

$$
m_{Y}(t)=\int_{-\infty}^{\infty} e^{t Y} \frac{1}{\sigma \sqrt{2 \pi}} e^{\frac{1}{2}\left(\frac{y-\mu}{\sigma}\right)^{2} d y}+\int_{-\infty}^{\infty} e^{t Y} \frac{1}{\sigma \sqrt{2 \pi}} e^{e^{\frac{1}{(2}\left(\frac{y-\mu}{\sigma}\right)^{2} d y}}
$$

Sin embargo, dado que,

$$
\frac{1}{1-F(a)} \int_{-\infty}^{\infty} e^{t Y} \frac{1}{\sigma \sqrt{2 \pi}} e^{\frac{1}{2}\left(\frac{y-\mu}{\sigma}\right)^{2} d y}
$$

es la función generadora de momentos de una variable aleatoria normal truncada a la izquierda de $y=a$, a la cual se le denotará como $m_{Y \mid Y>a}(t)$; es decir,

$$
m_{y \mid y>a(t)} \frac{1}{1-F(a)} \int_{-\infty}^{\infty} e^{t Y} \frac{1}{\sigma \sqrt{2 \pi}} e^{\left.\frac{1}{2\left(\frac{y-\mu}{\sigma}\right.}\right)^{2} d_{y}}
$$

al sustituir (A.2) y (A.4) en (A.3), se obtiene el siguiente resultado:

$$
e^{\mu t+\frac{1}{2} \sigma^{2} t^{2}}=\frac{1}{\sigma \sqrt{2 \pi}} e^{t y \frac{1}{2}\left(\frac{y-\mu}{\sigma}\right)^{2} d_{y}+[1-F(a)] m_{y \mid y>a}(t)}
$$

al completar el trinomio cuadrado perfecto en el exponente del integrando de la primera integral y después de simplificar, se alcanza el siguiente resultado:

$$
e^{\mu t+\frac{1}{2} \sigma^{2} t^{2}}=e^{\mu t+\frac{1}{2} \sigma^{2} t^{2}}\left\{\frac{1}{\sigma \sqrt{2 \pi}} \int_{-\infty}^{a} e^{\frac{1}{2}\left[\frac{y-\left(\mu+t \sigma^{2}\right)}{\sigma}\right]^{2}} d y\right\}+[1-F(a)] m_{y \mid y>a}(t)
$$

dado que el término que aparece entre llaves es igual a $\operatorname{Pr}(y \leq a)$ para una variable aleatoria normal con media $\mu+t \sigma^{2}$ y varianza $\sigma^{2}$; al estandarizar esta variable, el resultado anterior se puede rescribir como:

$$
e^{\mu t+\frac{1}{2} \sigma^{2} t^{2}}=e^{\mu t+\frac{1}{2} \sigma^{2} t^{2}} \Phi(\omega)+[1-F(a)] m_{y \mid y>a}(t)
$$


en donde $\Phi(\omega)$ denota a la función de distribución acumulada de una variable aleatoria normal estándar, con:

$$
\omega=\frac{a-\left(\mu+t a^{2}\right)}{\sigma}
$$

luego, se escribe $F(\alpha)$ como $\Phi(\alpha)$, para lo cual se toma en cuenta que:

$$
\alpha=\frac{a-\mu}{\sigma}
$$

Por último, al despejar $m_{y \mid y>a}(t)$, se obtiene el resultado deseado:

$$
m_{y \mid y>a}(t)=\frac{1-\Phi(\omega)}{1-\Phi(\alpha)} e^{\mu t+\frac{1}{2} \sigma^{2} t^{2}}
$$

La cual es la función generadora de momentos de una variable aleatoria normal truncada a la izquierda de $\mathrm{y}=\mathrm{a}$. De esta forma, se puede calcular la media y la varianza de esta variable aleatoria. ${ }^{11}$ Para ello simplemente se emplean las propiedades de dicha función generadora.

"La función generadora de momentos de una variable aleatoria normal, cuando el truncamiento se da por arriba del punto $y=a$, está dada por:

$$
m_{y \mid y>a}(t)=\frac{1-\Phi(\omega)}{1-\Phi(\alpha)} e^{\mu t+\frac{1}{2} \sigma^{2} t^{2}}
$$

La cual se obtiene de manera análoga a la función generadora de una variable aleatoria normal truncada a la izquierda del punto $y=a$. 
